\title{
Incidencia del buceo autónomo y capacidad de carga en sitios de buceo del Parque Nacional Jardines de la Reina, Cuba
}

\section{Scuba diving incidence and carrying capacity of dive sites at Jardines de la Reina National Park, Cuba}

\author{
Leslie Hernández Fernández ${ }^{*}$, Yunier M. Olivera Espinosa ${ }^{1}$, Tamara Figueredo Martín ${ }^{1}$, Raúl \\ Gómez Fernández ${ }^{1}$ Leander Brizuela Pardo ${ }^{1}$ y Fabián Pina Amargós ${ }^{l}$
}

\begin{abstract}
RESUMEN
Una de las actividades que más se desarrolla en el Parque Nacional Jardines de la Reina (PNJR), ubicado al sur de las provincias de Sancti Spíritus, Ciego de Ávila y Camagüey, es el buceo autónomo. Sobre esta base se realizó un estudio acerca de la incidencia de dicha actividad sobre los arrecifes de esta región, específicamente, sobre los corales pétreos. Para ello se estudiaron 28 sitios, en 10 días, lo que permitió comparar zonas de alta, media, baja y sin intensidad de buceo en cuanto al porcentaje de cobertura viva de los corales, mediante 220 transectos lineales y con la observación de 51 buceadores. En los sitios con alta intensidad de buceo se identificaron 36 especies de corales, siendo las más abundantes: $S$. siderea, $A$. agaricites, $P$. astreoides, $M$. alcicornis y $O$. faveolata. De los 51 buceadores observados, 48 cometieron infracciones: contactos sobre los organismos, resuspensión de sedimentos, y en menor medida, daños mecánicos (rompimiento). Las gorgonias fueron los organismos más contactados, seguidas por los corales. La cantidad de toques por buceador en el PNJR fue de 5.8 toques $/ \mathrm{min}$. La capacidad de carga efectiva en el PNJR varió entre 15 y 35 buceadores/sitio/día. Se infiere que los sitios de buceo del PNJR, a pesar de la incidencia del buceo autónomo sobre ellos, no muestran deterioro. No obstante, se recomienda monitorear los organismos afectados para conocer con precisión su evolución ante las incidencias, así como cumplir con el número de buceadores/sitio/día que se sugiere en este estudio.
\end{abstract}

Palabras claves: Buceo autónomo, corales pétreos, Parque Nacional Jardines de la Reina, capacidad de carga, incidencias del buceo.

\begin{abstract}
Scuba diving is one of the main activities in the Jardines de la Reina National Park (PNJR), located off the southern coast of Sancti Spiritus, Ciego de Avila and Camagüey provinces. This study was conducted to determine the effect of this activity on the coral reefs of the area, particularly on stony corals. A total of 28 sites were surveyed during a ten-day period. Areas with high, medium, and low diving intensity, as well as no diving were compared taking into account the percentage of stony coral cover. A total of 220 line transects were used and 51 divers were observed. In sites with high diving intensity, 36 species of stony corals were identified, mostly including $S$. siderea, $A$. agaricites, $P$. astreoides, $M$. alcicornis and $O$. faveolata. Of the 51 divers observed, 48 violated diving regulations such as making contact with the corals, sediment resuspension, and, to a lesser degree, mechanical damage (breaking of organisms). Gorgonians, followed by stony corals, were the most contacted organisms. The amount of touches per diver was 5.8 touches/minute. Effective carrying capacity in PNJR ranged from 15 to 35 divers/site/day. It is inferred that, in spite of the impact of scuba diving recorded on the surveyed sites, the sites showed no signs of deterioration. However, monitoring affected organisms to follow up post-damage evolution and complying with the number of divers/site/day suggested in this study is recommended.
\end{abstract}

Keywords: Scuba diving, stony corals, Jardines de la Reina National Park, carrying capacity, diving incidents.

Centro de Investigaciones de Ecosistemas Costeros (CIEC). Cayo Coco. Morón. CP: 69400. Ciego de Ávila. Cuba. leslie@ciec.cu*,yunier@ciec.cu, tamara@ciec.cu,raul@ciec.cu, leander@ciec.cu, fabian@ciec.cu

Recibido: 10 de setiembre de 2015

Corregido: 1 de febrero de 2016

Aceptado: 16 de febrero de 2016

DOI: http://dx.doi.org/10.15359/revmar.8-2.1

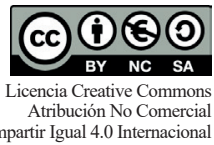

Rev. Mar. Cost. ISSN 1659-455X. Vol. 8 (2): 9-27, Julio-Diciembre 2016. 


\section{INTRODUCCIÓN}

El archipiélago de los Jardines de la Reina se encuentra ubicado al sur de las provincias de Sancti Spíritus, Ciego de Ávila y Camagüey, en la región central de Cuba. Está formado por 661 cayos y su extensión es de 135 $\mathrm{km}$, de los cuales alrededor de $950 \mathrm{~km}^{2}$ fueron declarados como Zona Bajo Régimen Especial de Uso y Protección (ZBREUP) en el año 1996, según la resolución ministerial de la Industria Pesquera 562/96 (Figueredo-Martín et al. 2010). Además, le fue otorgada, por el Comité Ejecutivo del Consejo de Ministros de Cuba, la categoría de Parque Nacional a la totalidad de su extensión, por acuerdo 6803/2010, debido a sus valores ecológicos y estado de conservación. Igualmente, Linton et al. (2002) lo consideraron entre las áreas menos dañadas del Caribe; Appeldoorn \& Lindeman (2003) lo calificaron entre las mayores reservas marinas de esta región.

En los ecosistemas relevantes del Parque Nacional Jardines de la Reina (PNJR), están los arrecifes coralinos, los que además de constituir zonas de refugio, alimentación, reproducción y crianza de numerosos organismos, proveen a las comunidades costeras de sustanciales beneficios económicos a través de actividades como la pesca y el turismo (McField \& Kramer, 2007; Miththapala, 2008; Figueredo-Martín et al. 2013; Figueredo-Martín et al. 2014a).

A pesar de ser el buceo autónomo una de las actividades que más se desarrolla en el PNJR, evaluado por los visitantes como excelente, superando las expectativas de aquellos que concurren al lugar y con un gran reconocimiento internacional (Figueredo-Martín et al. 2010), los trabajos científicos ejecutados en la zona han estado vinculados al conocimiento del estado general del arrecife y no a dicha actividad. Entre los estudios efectuados cabe destacar el realizado por Zlatarski \& MartínezEstalella (1980) sobre los corales pétreos, su taxonomía y fauna asociada a las colonias. Alcolado (1981) determinó la composición y estructura de las comunidades de octocorales y PinaAmargós et al. (2008) evaluaron su estructura y composición a lo largo del archipiélago, llegando a la conclusión que son relativamente homogéneas. Recientemente, Hernández-Fernández et al. (2011) actualizaron la composición de las comunidades de corales pétreos y de octocorales, y estudiaron la respuesta de estos organismos ante el evento de blanqueamiento.

Estudios, como los realizados por Zakai \& Chadwick-Furman, 2002; Barker \& Roberts, 2004; Álvarez, 2012 y Cederstav et al. 2015, exponen que el buceo autónomo puede ser una de las amenazas para los arrecifes coralinos. Se esperaría, por tanto, que en el PNJR los sitios con "intensidad alta del buceo" presenten condiciones menos favorables, para algunos indicadores ecológicos, como la cobertura, diversidad y densidad de corales pétreos, que aquellos donde la intensidad es baja o no se realiza esta actividad. Sobre esta hipótesis, este trabajo se propone determinar las incidencias del buceo autónomo 
sobre los arrecifes coralinos y la capacidad de carga de los sitios de buceo del PNJR, lo cual constituye una herramienta de planificación para sustentar las decisiones de manejo en esta Área Marina Protegida (AMP).

Debido a que el turismo en el PNJR aporta beneficios significativos, que incluyen la conservación del recurso y la generación de ingresos para actividades de conservación y manejo (FigueredoMartín et al. 2013; 2014a), es importante una planificación y una gestión adecuadas (Perera-Valderrama et al. 2007).

\section{MATERIALES Y MÉTODOS}

Área de estudio: El estudio se realizó en octubre de 2014 en el PNJR, el cual se extiende desde Cayo Bretón hasta Cayo Cabeza del Este, en la parte meridional de la Isla de Cuba (Fig. 1). Los arrecifes coralinos del PNJR, donde se realiza buceo autónomo, se ubican al sur de los cayos, donde existen crestas someras y escarpes poco profundos entre los $10 \mathrm{~m}$ y $30 \mathrm{~m}$ de profundidad. En las crestas predominan las especies: Millepora complanata (Lamarck, 1816), Acropora palmata (Lamarck, 1816) y Porites astreoides (Lamarck, 1816), y en los escarpes poco profundos predominan: Siderastrea siderea (Ellis \& Solander, 1786) y Agaricia agaricites (Linnaeus, 1758). Este estudio se ejecutó en los escarpes poco profundos.

\section{Condición biótica de los corales} pétreos: De los 28 sitios estudiados, a 22 se les clasificó teniendo en cuenta la frecuencia con que son visitados para la actividad de buceo autónomo. Aquellos sitios que son visitados todos los días

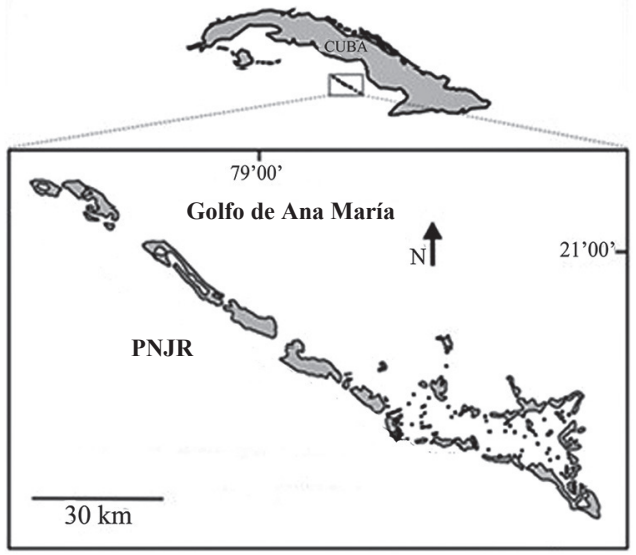

Fig. 1. Zona de estudio. PNJR: Parque Nacional Jardines de la Reina

Fig. 1. Study area. PNJR: Jardines de la Reina National Park

de la semana, se clasificaron como con "intensidad alta del buceo", los visitados una vez a la semana, como "intensidad media del buceo", los visitados con menor frecuencia, como "intensidad baja del buceo" y los sitios que no son visitados por los buceadores se clasificaron como "sin intensidad de buceo" (Cuadro 1). Esta selección se basó en entrevistas semiestructuradas realizadas al $50 \%$ de los instructores de buceo del área (de un total de 10 instructores) e informaciones obtenidas del monitoreo de la actividad de buceo autónomo, desarrollado en la zona por la operación turística. A estos 22 sitios se les analizó la condición biótica de los corales pétreos (cubrimiento, diversidad y densidad). Los restantes seis sitios se incluyeron en el cálculo de la capacidad de carga, y a tres de estos, también se les determinó las incidencias del buceo autónomo. A estos seis últimos sitios se les nombró como "otros sitios" (Cuadro 1). 
Cuadro 1. Sitios clasificados según la intensidad del buceo y donde se analizó la condición biótica de los corales pétreos y la capacidad de carga. Sitios donde se determinó la incidencia del buceo

Table 1 . Sites ranked by the diving intensity and where the biotic condition of stony corals and carrying capacity were analyzed. Sites where the incidence of diving was determined

\begin{tabular}{|c|c|c|c|c|}
\hline Sitios de buceo & Coordenadas & $\begin{array}{l}\text { Profundidad } \\
\text { (m) }\end{array}$ & $\begin{array}{l}\text { Región a la que } \\
\text { pertenecen }\end{array}$ & $\begin{array}{l}\text { Número de } \\
\text { buceadores }\end{array}$ \\
\hline \multicolumn{5}{|c|}{ Intensidad Alta del buceo (A) } \\
\hline 1-Cueva del Pulpo & $20^{\circ} 45^{\prime} 13.2^{\prime \prime}-78^{\circ} 50^{\prime} 14.1 "$ & 8 & Boca de Piedra & 8 \\
\hline 2-Five Sea & $20^{\circ} 45^{\prime} 38.4^{\prime \prime}-78^{\circ} 50^{\prime} 48.4^{\prime \prime}$ & 9 & Boca de Piedra & 10 \\
\hline 3-Cabezo de la Cubera & $20^{\circ} 45^{\prime} 34.6^{\prime \prime}-78^{\circ} 50^{\prime} 44.6^{\prime \prime}$ & 9 & Boca de Piedra & 9 \\
\hline 4-Mogotes & $20^{\circ} 45^{\prime} 43.6^{\prime \prime}-78^{\circ} 51^{\prime} 13.86^{\prime \prime}$ & 10 & Boca de Piedra & 10 \\
\hline 5-Pipín & $20^{\circ} 49^{\prime} 33.1^{\prime \prime}-78^{\circ} 58^{\prime} 50.0^{\prime \prime}$ & 15 & Caballones & \\
\hline 6-Farallón & $20^{\circ} 49^{\prime} 35.88^{\prime \prime}-78^{\circ} 59^{\prime} 54^{\prime \prime}$ & 17 & Caballones & 10 \\
\hline 7-Finca de Pepe & $20^{\circ} 47^{\prime} 20.7^{\prime \prime}-78^{\circ} 57^{\prime} 01.6 ”$ & 8 & Anclita & 10 \\
\hline 8-Anclita & $20^{\circ} 47^{\prime} 7.6^{\prime \prime} \quad-78^{\circ} 56^{\prime} 15.3^{\prime \prime}$ & 9 & Anclita & \\
\hline 9-Boca de Anclita & $20^{\circ} 47^{\prime} 13.3^{\prime \prime}-78^{\circ} 56^{\prime} 33.6^{\prime \prime}$ & 9 & Anclita & 10 \\
\hline \multicolumn{5}{|c|}{ Intensidad Media del buceo (M) } \\
\hline 10-Patricia Reef & $20^{\circ} 46^{\prime} 30.8^{\prime \prime}-78^{\circ} 55^{\prime} 07.9^{\prime \prime}$ & 9 & Anclita & 8 \\
\hline 11-La Cana & $20^{\circ} 46^{\prime} 28.0^{\prime \prime}-78^{\circ} 54^{\prime} 54.9^{\prime \prime}$ & 10 & Anclita & 10 \\
\hline 12-Pew Reef & $20^{\circ} 46^{\prime} 34.3^{\prime \prime}-78^{\circ} 55^{\prime} 21.2^{\prime \prime}$ & 10 & Anclita & 8 \\
\hline 13-La Raya & $78^{\circ} 54^{\prime} 54.9^{\prime \prime}-78^{\circ} 50^{\prime} 20.4^{\prime \prime}$ & 9 & Boca de Piedra & \\
\hline \multicolumn{5}{|c|}{ Intensidad Baja del buceo (B) } \\
\hline 14-Puente Escondido & $20^{\circ} 51^{\prime} 17.8^{\prime \prime}-79^{\circ} 02^{\prime} 55.1^{\prime \prime}$ & 10 & Caballones & 9 \\
\hline 15-Tampico Reef & $20^{\circ} 50^{\prime} 58.6^{\prime \prime}-79^{\circ} 02^{\prime} 12.3^{\prime \prime}$ & 9 & Caballones & \\
\hline 16-Luisa Reef & $20^{\circ} 45^{\prime} 04.3 ”-78^{\circ} 50^{\prime} 03.6 ”$ & 8 & Boca de Piedra & 10 \\
\hline 17-Pequeño Paraíso & $20^{\circ} 43^{\prime} 42.0^{\prime \prime}-78^{\circ} 48^{\prime} 39.3 \prime$ & 9 & Boca de Piedra & 8 \\
\hline 18-Cruces & $20^{\circ} 42^{\prime} 45.6^{\prime \prime}-78^{\circ} 47^{\prime} 16.5^{\prime \prime}$ & 10 & Las Cruces & \\
\hline 19-Crucecitas & $20^{\circ} 43^{\prime} 80.0^{\prime \prime}-78^{\circ} 47^{\prime} 58.1 ”$ & 10 & Las Cruces & 8 \\
\hline \multicolumn{5}{|c|}{ Sin intensidad de buceo (SB) } \\
\hline 20-Laberinto del Dátil & $20^{\circ} 52^{\prime} 33.0^{\prime \prime}-78^{\circ} 56^{\prime} 15.3^{\prime \prime}$ & 8 & Cayo Grande & \\
\hline 21-Este de Luisa & $20^{\circ} 44^{\prime} 41.9^{\prime \prime}-78^{\circ} 49^{\prime} 37.9^{\prime \prime}$ & 10 & Boca de Piedra & \\
\hline 22-Los Indios & $20^{\circ} 45^{\prime} 33.7^{\prime \prime}-78^{\circ} 51^{\prime} 12.0^{\prime \prime}$ & 22 & Boca de Piedra & \\
\hline \multicolumn{5}{|c|}{ Otros Sitios } \\
\hline$\overline{23-C o r a l ~ N e g r o ~} 1$ & $20^{\circ} 45^{\prime} 8.1^{\prime \prime}-78^{\circ} 50^{\prime} 28.2^{\prime \prime}$ & 28 & Boca de Piedra & 9 \\
\hline 24-Coral Negro 2 & $20^{\circ} 45^{\prime} 02.2^{\prime \prime}-78^{\circ} 50^{\prime} 18.2^{\prime \prime}$ & 22 & Boca de Piedra & 10 \\
\hline 25-El Peruano & $20^{\circ} 50^{\prime} 39.5^{\prime \prime}-79^{\circ} 01^{\prime} 17.7 "$ & 9 & Caballones & 10 \\
\hline 26-Los Pinos & $20^{\circ} 50^{\prime} 37.4^{\prime \prime}-79^{\circ} 00^{\prime} 35.8^{\prime \prime}$ & 9 & Caballones & \\
\hline 27-Los Sábalos & $20^{\circ} 47^{\prime} 34.1 "$ - $78^{\circ} 57^{\prime} 03.8^{\prime \prime}$ & 10 & Anclita & \\
\hline 28-El Galeón & $20^{\circ} 47^{\prime} 46.1 "$ - $78^{\circ} 57^{\prime} 10.0^{\prime \prime}$ & 10 & Anclita & \\
\hline
\end{tabular}

Para conocer la condición biótica de los corales pétreos se tuvo en cuenta la metodología propuesta por Caballero et al. (2013), como versión ajustada de AGRRA (2000). En cada sitio de buceo fue extendida una cinta métrica delimitando $10 \mathrm{~m}$ de largo, justo sobre la superficie del arrecife (transecto), 
hasta completar $100 \mathrm{~m}$. A cada colonia que quedó debajo del transecto se le determinó, in situ, la especie, se le cuantificaron la densidad (colonia/10 m) y el cubrimiento vivo. Se analizaron, en total, 220 transectos lineales y se contaron 7168 colonias de corales pétreos. Para la identificación de los órdenes Milleporina y Scleractinia se siguieron los criterios de Zlatarski \& Martínez-Estalella, 1980 y González-Ferrer, 2009.

Para conocer si existían diferencias significativas entre las zonas de buceo, para el cubrimiento de coral vivo (\%), se realizó un análisis de varianza bifactorial según Zar (1996), donde se consideró como factor 1, el cubrimiento de coral vivo y como factor 2 , las zonas. Se realizaron varias combinaciones de sitios, como diseño aleatorio estratificado, donde entre las distintas intensidades se escogieron, al azar, los sitios y se consideraron como estratos las diferentes intensidades de buceo. Previo al análisis se determinaron la media, la varianza y el coeficiente de variación para conocer la distribución de los datos, con el fin de garantizar la homogeneidad de varianza y la normalidad, las que fueron comprobadas por la prueba de Bartlett y Kolmogorov-Smirnov, respectivamente, todas según Zar (1966). Los gráficos y cálculos de análisis de varianza se ejecutaron con el programa STATISTICA 7 (StatSoft, 2004).

Incidencias del buceo autónomo: Para determinar las incidencias del buceo autónomo sobre los arrecifes coralinos del PNJR, se tuvo en cuenta la metodología aplicada por HernándezFernández et al. (2008) para los arrecifes de Cayo Coco y recomendada por
Figueredo-Martín et al. (2014b) para las APMs de Cuba. Se monitorearon 51 buceadores (32\% del total), en 17 sitios de buceo diferentes (3 buceadores por sitios), en profundidades que oscilaron entre los $8 \mathrm{~m}$ y $28 \mathrm{~m}$ (Cuadro 1). Cada buceador monitoreado se observó durante $10 \mathrm{~min}$. El buceador no percibió que estaba siendo monitoreado. Se tuvo en cuenta el género (masculino y femenino). Se definieron como adultos los mayores de 30 años, como jóvenes aquellos cuya edad osciló entre 15 y 30 años, y como menores, los menores de 15 años. Se tuvo en cuenta, además, si buceaban con cámara subacuática o no. Todos los buceadores observados tenían sus certificaciones correspondientes, aunque no se tuvo en cuenta el número de buceos que realmente habían realizado, lo que demuestra si tienen experiencia o no.

Durante los 10 min. que se observó a un buceador, se anotó en una tablilla si cometía alguna infracción relacionada con recolectas, con daños mecánicos (rompimientos), si contactaba algún organismo, se paraba sobre el arrecife o vertía basura. Se tuvo en cuenta qué organismos recibían las infracciones, fundamentalmente, si eran esponjas, gorgonias o corales pétreos, así como otros organismos bentónicos. También se anotaba la parte del equipo de buceo o del cuerpo del buceador, con la cual se cometía la infracción.

Capacidad de carga: El cálculo de capacidad de carga se realizó para 27 sitios de buceo (Cuadro 2), empleando la metodología de Cifuentes et al. (1999), adaptada por Gallo et al. (2001) y Sousa- 
Cuadro 2. Otras variables que se tuvieron en cuenta para el cálculo de la capacidad de carga por sitios de buceo

Table 2. Other variables taken into account to calculate carrying capacity per dive site

\begin{tabular}{|c|c|c|c|}
\hline Sitios de buceo & $\begin{array}{l}\text { Longitud del } \\
\text { recorrido }(\mathrm{m})\end{array}$ & $\begin{array}{c}\text { Tiempo del } \\
\text { recorrido }(\mathrm{min})\end{array}$ & $\begin{array}{l}\% \text { de corales } \\
\text { ramificados }\end{array}$ \\
\hline Anclita & 200 & 50 & 21 \\
\hline Boca de Anclita & 200 & 50 & 10.9 \\
\hline Los Mogotes & 200 & 50 & 15.1 \\
\hline Laberinto del Dátil & 200 & 50 & 17.8 \\
\hline Puente Escondido & 200 & 50 & 10.1 \\
\hline Pew Reef & 500 & 50 & 12.7 \\
\hline Las Cruces & 200 & 50 & 11 \\
\hline La Crucecita & 200 & 50 & 12.8 \\
\hline La Cana & 500 & 50 & 13.7 \\
\hline Los Indios & 50 & 25 & 19.8 \\
\hline Cabezo de la Cubera & 200 & 50 & 26.8 \\
\hline Luisa Reef & 200 & 50 & 21.4 \\
\hline Cueva del Pulpo & 200 & 50 & 10.9 \\
\hline Coral Negro 2 & 50 & 25 & 5 \\
\hline Pequeño Paraíso & 200 & 50 & 12.8 \\
\hline Finca de Pepe & 200 & 50 & 23.1 \\
\hline Farallón & 200 & 50 & 24 \\
\hline Tampico Reef & 200 & 50 & 20.8 \\
\hline El Peruano & 200 & 50 & 13.3 \\
\hline Pipín & 200 & 50 & 23.6 \\
\hline Los Pinos & 200 & 50 & 7.4 \\
\hline Coral Negro 1 & 50 & 25 & 0 \\
\hline La Raya & 200 & 50 & 10.1 \\
\hline Five Sea & 200 & 50 & 19.3 \\
\hline Patricia Reef & 500 & 50 & 14.1 \\
\hline Los Sábalos & 200 & 50 & 19.8 \\
\hline El Galeón & 200 & 50 & 8.4 \\
\hline
\end{tabular}

Melo et al. (2006). Dicha metodología consta de tres etapas, en las que se incluyen condiciones físicas, biológicas y de manejo, presentes en el PNJR (Cuadro 3). Se tuvieron en cuenta también: la longitud del recorrido de cada buceo, el tiempo del recorrido y el porcentaje de corales ramificados (Cuadro 2). Los factores de corrección que se emplearon fueron: factor de corrección por fragilidad ( $\mathrm{FCfg}$ ), factor de corrección por toques (FCtq), factor de corrección por condiciones meteorológicas (FCmet) y factor de corrección social (FCsoc), así como la capacidad de manejo (CM) (Cuadro 4).

\section{RESULTADOS}

Condición de los corales pétreos:

Entre los sitios con alta intensidad de buceo se identificaron 36 especies 
Cuadro 3. Variables que se tuvieron en cuenta para el cálculo de la capacidad de carga por sitios de buceo

Table 3. Variables taken into account to calculate carrying capacity per dive site

\begin{tabular}{|c|c|c|}
\hline Variables & Valor & Comentarios \\
\hline $\begin{array}{l}\text { Número de buceadores por } \\
\text { grupos }\end{array}$ & 10 & $\begin{array}{l}\text { Ocho buceadores como promedio por grupo y un instructor } \\
\text { (guía) por cada seis buceadores. }\end{array}$ \\
\hline $\begin{array}{l}\text { Número de grupos por } \\
\text { sitios de buceo }\end{array}$ & 1 & Un solo grupo está en un sitio de buceo dado a la vez. \\
\hline $\begin{array}{l}\text { Longitud de un buceador } \\
\text { (m) }\end{array}$ & 4 & $\begin{array}{l}2 \mathrm{~m} \text { un buceador, incluyendo las aletas. } 2 \mathrm{~m} \text { de distancia } \\
\text { entre parejas de buceadores. }\end{array}$ \\
\hline $\begin{array}{l}\text { Distancia ocupada por un } \\
\text { grupo de buceadores }\end{array}$ & $\mathrm{L}$ & Igual al recorrido que se realiza en cada sitio de buceo. \\
\hline $\begin{array}{l}\text { Tiempo disponible de } \\
\text { buceo por día (min) }\end{array}$ & 540 & $\begin{array}{l}\text { Se tiene disponible prácticamente todo el día, desde las } \\
\text { 08:00 a.m. hasta las 05:00 p.m. }\end{array}$ \\
\hline $\begin{array}{l}\text { Horas de cierre al año por } \\
\text { condiciones climáticas } \\
\text { adversas }\end{array}$ & 54 & $\begin{array}{l}\text { Cuando prevalecen eventos meteorológicos extremos se } \\
\text { buscan otras alternativas, no vinculadas al buceo. Se pierden } \\
\text { alrededor de } 6 \text { días al año. }\end{array}$ \\
\hline Horas de trabajo al año & 2223 & $\begin{array}{l}\text { Los sitios de buceo están abiertos } 247 \text { días al año, por } 9 \text { horas } \\
\text { disponibles para el buceo por día. Los restantes días están } \\
\text { dedicados al transporte del personal y al mantenimiento de } \\
\text { las instalaciones que permanecen en el Parque Nacional } \\
\text { Jardines de la Reina. }\end{array}$ \\
\hline $\begin{array}{l}\text { Frecuencia de toques por } \\
\text { buceadores por minuto }\end{array}$ & 5.8 & \\
\hline $\begin{array}{l}\text { Capacidad de manejo } \\
\text { relativa a la infraestructura }\end{array}$ & 0.85 & \\
\hline $\begin{array}{l}\text { Capacidad de manejo } \\
\text { relativa a equipamiento }\end{array}$ & 0.83 & \\
\hline $\begin{array}{l}\text { Capacidad de manejo } \\
\text { relativa al personal }\end{array}$ & 0.97 & \\
\hline
\end{tabular}

de corales pétreos, siendo las más abundantes: $S$. siderea, A. agaricites, P. astreoides, Millepora alcicornis (Linnaeus, 1758) y Orbicella faveolata (Ellis \& Solander, 1786). Hubo mayor diversidad de corales en los sitios Five Sea y Pipín, y mayor densidad en Five Sea y en Cueva del Pulpo (Cuadro 5).

En los sitios de las zonas con media y baja intensidad de buceo se identificaron 35 especies de corales pétreos. Las especies más abundantes coinciden con las encontradas en los sitios de la zona con alta intensidad de buceo. El sitio de mayor diversidad y densidad de corales fue La Raya, mientras que el de menor diversidad y densidad fue La Cana (Cuadro 5). En los sitios sin intensidad de buceo se identificaron 22 especies de corales pétreos. Las especies más abundantes fueron: A. agaricites, $O$. faveolata y $M$. alcicornis. El sitio de mayor diversidad de corales fue Los Indios y el de mayor densidad, Laberinto del Dátil (Cuadro 5). 
Cuadro 4. Número de veces que podría repetirse la visita, al día, a un sitio de buceo $(\mathrm{Nv})$. Factor de corrección por fragilidad (FCfg), factor de corrección por toques (FCtq), factor de corrección por condiciones meteorológicas (FCmet) y factor de corrección social (FCsoc). Capacidad de manejo (CM)

Table 4. Number of times a diving place (Nv) could be repeatedly visited per day. Correction factor for fragility (FCfg), correction factor for touches (FCtq), correction factor for meteorological conditions (FCmet) and social correction factor (FCsoc). Handling capacity (CM)

\begin{tabular}{lcccccc}
\hline \multicolumn{1}{c}{ Sitios de buceo } & Nv & FCfg & FCtq & FCmet & FCsoc & CM \\
\hline Anclita & 10.8 & 0.79 & 0.88 & 0.98 & 0.05 & 0.88 \\
Boca de Anclita & 10.8 & 0.89 & 0.88 & 0.98 & 0.05 & 0.88 \\
Los Mogotes & 10.8 & 0.85 & 0.88 & 0.98 & 0.05 & 0.88 \\
Laberinto del Dátil & 10.8 & 0.82 & 0.88 & 0.98 & 0.05 & 0.88 \\
Puente Escondido & 10.8 & 0.90 & 0.88 & 0.98 & 0.05 & 0.88 \\
Pew Reef & 10.8 & 0.87 & 0.88 & 0.98 & 0.02 & 0.88 \\
Las Cruces & 10.8 & 0.89 & 0.88 & 0.98 & 0.05 & 0.88 \\
La Crucecita & 10.8 & 0.87 & 0.88 & 0.98 & 0.05 & 0.88 \\
La Cana & 10.8 & 0.86 & 0.88 & 0.98 & 0.02 & 0.88 \\
Los Indios & 21.6 & 0.80 & 0.77 & 0.98 & 0.2 & 0.88 \\
Cabezo de la Cubera & 10.8 & 0.73 & 0.88 & 0.98 & 0.05 & 0.88 \\
Luisa Reef & 10.8 & 0.79 & 0.88 & 0.98 & 0.05 & 0.88 \\
Cueva del Pulpo & 10.8 & 0.89 & 0.88 & 0.98 & 0.05 & 0.88 \\
Coral Negro 2 & 21.6 & 0.95 & 0.77 & 0.98 & 0.2 & 0.88 \\
Pequeño Paraíso & 10.8 & 0.87 & 0.88 & 0.98 & 0.05 & 0.88 \\
Finca de Pepe & 10.8 & 0.77 & 0.88 & 0.98 & 0.05 & 0.88 \\
Farallón & 10.8 & 0.76 & 0.88 & 0.98 & 0.05 & 0.88 \\
Tampico Reef & 10.8 & 0.79 & 0.88 & 0.98 & 0.05 & 0.88 \\
El Peruano & 10.8 & 0.87 & 0.88 & 0.98 & 0.05 & 0.88 \\
Pipín & 10.8 & 0.76 & 0.88 & 0.98 & 0.05 & 0.88 \\
Los Pinos & 10.8 & 0.93 & 0.88 & 0.98 & 0.05 & 0.88 \\
Coral Negro 1 & 21.6 & 1 & 0.77 & 0.98 & 0.2 & 0.88 \\
La Raya & 10.8 & 0.90 & 0.88 & 0.98 & 0.05 & 0.88 \\
Five Sea & 10.8 & 0.81 & 0.88 & 0.98 & 0.05 & 0.88 \\
Patricia Reef & 10.8 & 0.86 & 0.88 & 0.98 & 0.02 & 0.88 \\
Los Sábalos & 10.8 & 0.80 & 0.88 & 0.98 & 0.05 & 0.88 \\
El Galeón & 10.8 & 0.92 & 0.88 & 0.98 & 0.05 & 0.88 \\
\hline
\end{tabular}

Al comparar las zonas de diferente intensidad de buceo, con varias combinaciones de sitios, se observó que los sitios sin buceo son los de menor cobertura de corales vivos. De las seis combinaciones analizadas (Fig. 2), en cinco, la zona de mayor intensidad de buceo mostró mayor cobertura (paneles A, B, C, D, E, menor cobertura en F, F: 10.9 y $P: 0.000$ ); en cinco, la zona de intensidad media de buceo mostró mayor cobertura (paneles A, B, C, E, 
Cuadro 5. Total de colonias (colonias), especies (especies), densidad (colonias/10 m) y porcentaje de cubrimiento (\% cubrimiento) en los sitios con alta intensidad de buceo, con media y baja intensidad de buceo y sitios sin intensidad de buceo

Table 5. Total colonies (colonies), species (species), density (colony/10 m) and percentage of coverage ( $\%$ coverage) in sites with high intensity diving, with medium and low intensity diving, and sites without diving activity

\begin{tabular}{|c|c|c|c|c|}
\hline Sitios & Colonias & Especies & Colonias/10 m & $\%$ Cubrimiento \\
\hline \multicolumn{5}{|c|}{ Sitios con alta intensidad de buceo } \\
\hline Cueva del Pulpo & 218 & 14 & 39.5 & 26 \\
\hline Five Sea & 310 & 26 & 44.1 & 30 \\
\hline Cabezo de la Cubera & 261 & 19 & 33.0 & 21 \\
\hline Mogotes & 238 & 22 & 31.9 & 25 \\
\hline Pipín & 250 & 26 & 31.6 & 21 \\
\hline Farallón & 220 & 22 & 27.0 & 19 \\
\hline Finca de Pepe & 255 & 21 & 32.0 & 20 \\
\hline Anclita & 187 & 19 & 20.7 & 18 \\
\hline Boca de Anclita & 165 & 15 & 19.2 & 14 \\
\hline \multicolumn{5}{|c|}{ Sitios con media y baja intensidad de buceo } \\
\hline Patricia Reef & 268 & 21 & 33.6 & 20 \\
\hline La Cana & 218 & 14 & 26.1 & 16 \\
\hline Pew Reef & 266 & 19 & 34.3 & 22 \\
\hline La Raya & 314 & 27 & 45.9 & 32 \\
\hline P. Escondido & 247 & 21 & 30.3 & 18 \\
\hline Tampico Reef & 268 & 23 & 34.9 & 23 \\
\hline Luisa Reef & 224 & 18 & 27.1 & 17 \\
\hline P. Paraíso & 264 & 23 & 36.0 & 27 \\
\hline Cruces & 271 & 17 & 35.6 & 24 \\
\hline Crucecitas & 257 & 20 & 33.1 & 22 \\
\hline \multicolumn{5}{|c|}{ Sitios sin intensidad de buceo } \\
\hline Laberinto del Dátil & 252 & 20 & 30.8 & 18 \\
\hline Este de Luisa & 206 & 18 & 25.1 & 18 \\
\hline Los Indios & 136 & 21 & 20.3 & 13 \\
\hline
\end{tabular}

F, menor cobertura en D, F: 11.3 y $P$ : 0.000 ); en cuatro, la zona de menor intensidad de buceo mostró mayor cobertura (paneles B, C, E, F, menor cobertura en A, F: 4.91 y $P: 0.008$; y D, F: 11.3 y $P: 0.000)$. En ninguno de los tres casos donde se incluyó la zona sin buceo, esta mostró mayor cobertura de coral vivo (menores coberturas en D, F: 11.3 y $P: 0.000 ;$ E, F: 5.98 y $P: 0.000$ y
F, F: 10.9 y $P: 0.000)$. Estos resultados muestran que los sitios donde existe una intensidad alta de buceo no presentan condiciones menos favorables, para la cobertura de corales pétreos, que aquellos donde la intensidad es media, baja o no se realiza esta actividad. Tampoco muestran condiciones menos favorables en cuanto a los índices ecológicos de diversidad y densidad. 


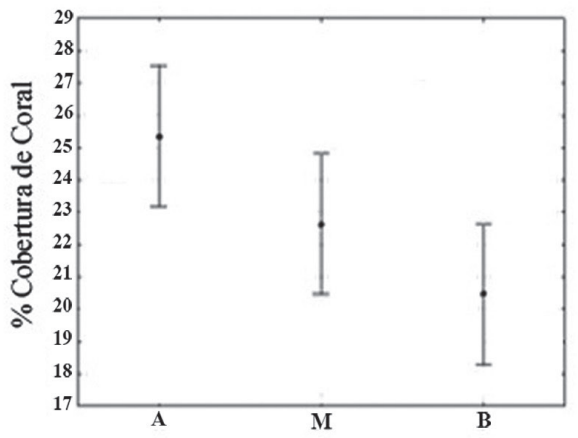

$\mathbf{A}$



C

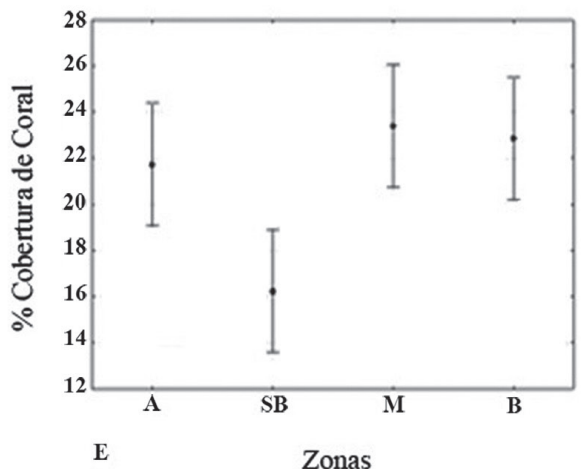

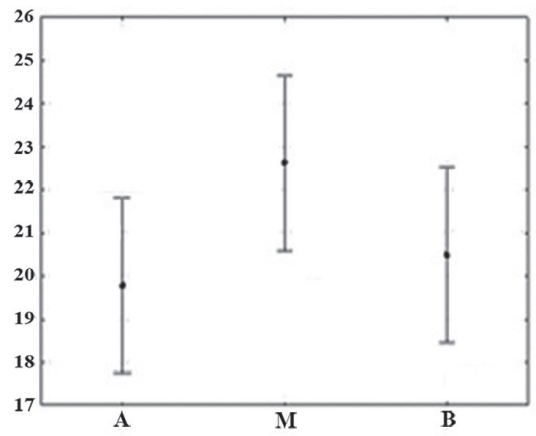

B

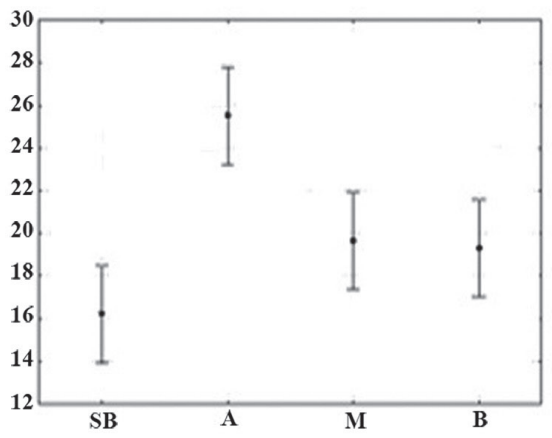

D

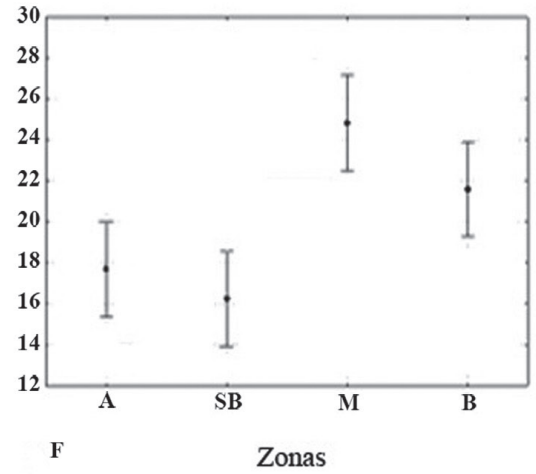

Fig. 2. Comparaciones entre zonas con diferente intensidad de buceo (los paneles muestran las comparaciones entre zonas con diferente intensidad de buceo a partir de varias combinaciones de sitios de buceo). A. Porcentaje de cubrimiento de coral vivo entre las zonas de alta (A) (1, 2, 3 y 4), media (M) (10, 11, 12 y 13) y baja intensidad de buceo (B) (14, 19, 16 y 18). B. Porcentaje de cubrimiento de coral vivo entre las zonas de alta (A) $(1,3,6$ y 8$)$, media (M) $(10,11,12$ y 13$)$ y baja intensidad de buceo (B) $(14,19,16$ y 18). C. Porcentaje de cubrimiento de coral vivo entre las zonas de alta (A) (1, 3, 6 y 9), media (M) (10, 11, 12 y 13) y baja intensidad de buceo (B) (15, 17,16 y 14). D. Porcentaje de cubrimiento de coral vivo entre las zonas sin buceo (SB) $(22,21$ y 
20), de alta intensidad de buceo (A) (1, 3 y 6), media (M) (10, 11 y 12) y baja (B) (15, 17 y 16). E. Porcentaje de cubrimiento de coral vivo entre las zonas de alta intensidad de buceo (A) $(4,5$ y 6), sin buceo (SB) (22, 21 y 20), media (M) (11, 12 y 13) y baja (B) (15, 17 y 14). F. Porcentaje de cubrimiento de coral vivo entre las zonas de alta intensidad de buceo (A) (7, 8 y 9), sin buceo (SB) $(22,21$ y 20$)$, media (M) (10, 11 y 12) y baja (B) $(18,15$ y 14)

Fig. 2. Comparisons between areas with different diving intensity (panels show comparisons between areas with different diving intensities from various combinations of dive sites). A. Alive coral cover percentage between areas of high (A) (1, 2, 3 and 4), medium (M) (10, 11, 12 and $13)$ and low diving intensity (B) $(14,19,16$ and 18$)$. B. Alive coral cover percentage between areas of high (A) (1, 3, 6 y 8), medium (M) (10, 11, 12 and 13) and low diving intensity (B) (14, 19,16 and 18). C. Alive coral cover percentage between areas of high (A) (1, 3, 6 \& 9), medium (M) $(10,11,12$ and 13) and low diving intensity (B) $(15,17,16$ and 14). D. Alive coral cover percentage between areas without diving activity (SB) $(22,21$ and 20). with high (A) $(1,3$ and 6$)$, medium (M) (10, 11 and 12) and low diving intensity (B) (15, 17 and 16). E. Alive coral cover percentage between areas of high (A) (4, 5 and 6), medium (M) (11, 12 and 13) and low diving intensity (B) (15, 17 and 14) and without diving activity (SB) (22, 21 and 20), F. Alive coral cover percentage between areas of high (A) (7, 8 and 9), medium (M) (10,11 and 12), low diving intensity (B) $(18,15$ and 14) and without diving activity (SB) $(22,21$ and 20)

Incidencias del buceo recreativo:

Se observó un total de 51 buceadores (Cuadro 6).

De estos, la mayoría (92\%) cometió infracciones (un mismo buceador cometió más de una infracción) vinculadas a contactos con los organismos (90\% de los buceadores realizaron contactos), seguida de la resuspensión de sedimentos $(47 \%$ de los buceadores incidieron sobre el sedimento), y en menor medida, en daños mecánicos (rompimiento) (37\% de los buceadores cometieron daños mecánicos). Las gorgonias fueron los organismos más contactados, seguidas por los corales pétreos. La parte del equipo de buceo con la que más infracciones se cometieron fue con las aletas (Fig. 3). Solo el 16\% de las 299 infracciones fueron cometidas por mujeres. La cantidad de toques por buceador en el PNJR fue de 5.8 toques/min., distribuida en 0.8 daños mecánicos/min. (rompimiento), 1.3 resuspensión de sedimentos/min. y 3.7 contactos $/ \mathrm{min}$. La mayoría de las infracciones cometidas fueron intencionales, provocadas por los buceadores que portaban cámaras subacuáticas y el mayor número de los contactos se produjo en los primeros 10 min., disminuyendo en la medida que iba avanzando el tiempo de buceo (E. del Sol, comunicación personal, octubre 22, 2014).

Capacidad de carga: La capacidad de carga efectiva (CCE) en el PNJR osciló entre 15 y 35 buceadores/sitio/día, siendo Cabezo de la Cubera el sitio de menor capacidad, mientras que el de mayor capacidad fue Coral Negro 1. El promedio total de buceadores/sitio/día en el PNJR fue de 19 (Fig. 4) y el anual de 4949. 


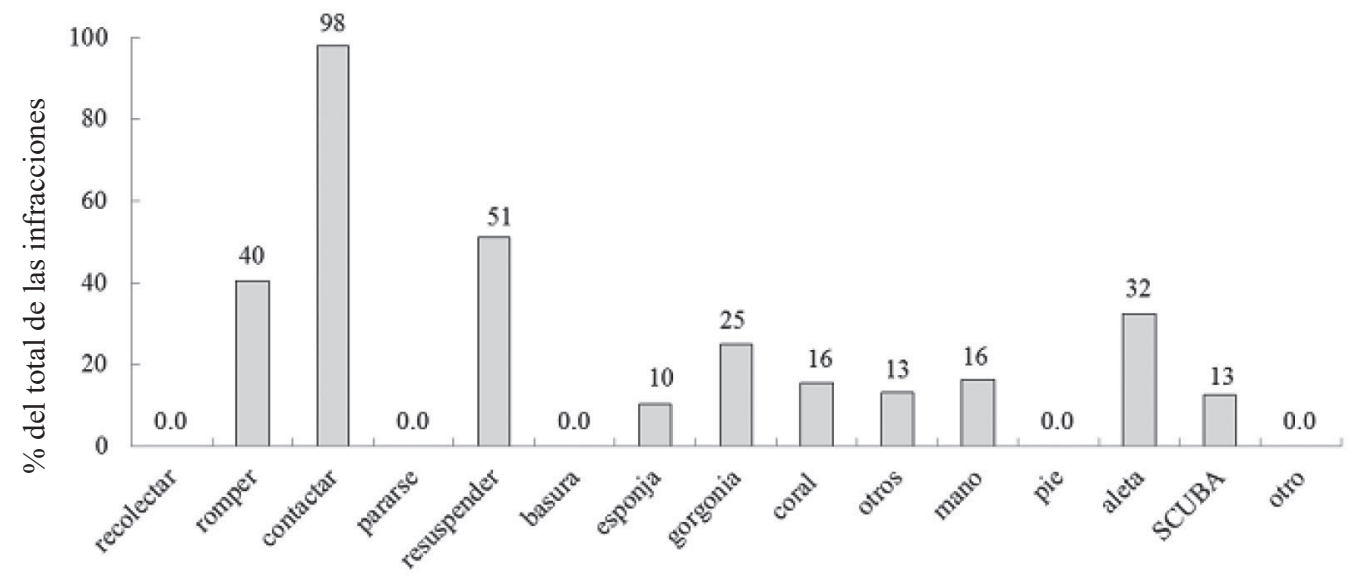

Fig. 3. Porcentaje total de infracciones. Tipo de infracción, organismos afectados por dichas infracciones y parte del equipo de buceo con que cometieron las infracciones $(\mathrm{N}=299)$ (SCUBA se utiliza para designar al equipo de buceo)

Fig. 3. Percentage of total infractions. Type of infraction, organism affected and part of diving gear causing the infraction $(\mathrm{N}=299)$ (SCUBA is used to designate the diving gear)

Cuadro 6. Datos generales de los buceadores observados

Table 6. General information on the observed divers

\begin{tabular}{cccccc}
\hline Datos generales & Adultos & Jóvenes & Masculino & Femenino & $\begin{array}{c}\text { Portadores } \\
\text { de cámaras } \\
\text { fotográficas }\end{array}$ \\
\hline \% de representación & 29 & 8 & 78 & 22 & 29 \\
\hline
\end{tabular}

\section{DISCUSIÓN}

El cubrimiento medio de coral vivo en los sitios con alta intensidad de buceo (25\%) fue similar al obtenido en la zona de buceo de María la Gorda en los hábitats de camellones y cabezos, el cual fue considerado bajo, según expuso De la Guardia et al. (2004). Un cubrimiento medio de coral vivo bajo fue considerado común para la mayoría de los arrecifes de Cuba y el Caribe (Wilkinson, 2000; Kramer, 2003).

Sin embargo, siguiendo los criterios de Alcolado \& Durán (2011), ante las escalas para la clasificación y puntaje de condición del bentos de arrecifes coralinos de Cuba y del Gran Caribe, para el cubrimiento vivo de corales (\%) (crítica: <5.0, pobre: 5.0-9.9, regular: 10.0-19.9, buena: 20.0-40.0 y muy buena: >40.0) se pudo constatar que la mayoría de los sitios de la zona con alta intensidad de buceo (6 de 9 sitios, o sea, el 67\%), manifestó una condición "Buena", ante este indicador ecológico, el resto manifestó una condición "Regular". En cuanto a la diversidad (número de especies de corales/10 m) (crítica: $<5.0$, pobre: 6-10, regular: 11-15, buena: 1620 y muy buena: $>20$ ), la mayoría de 


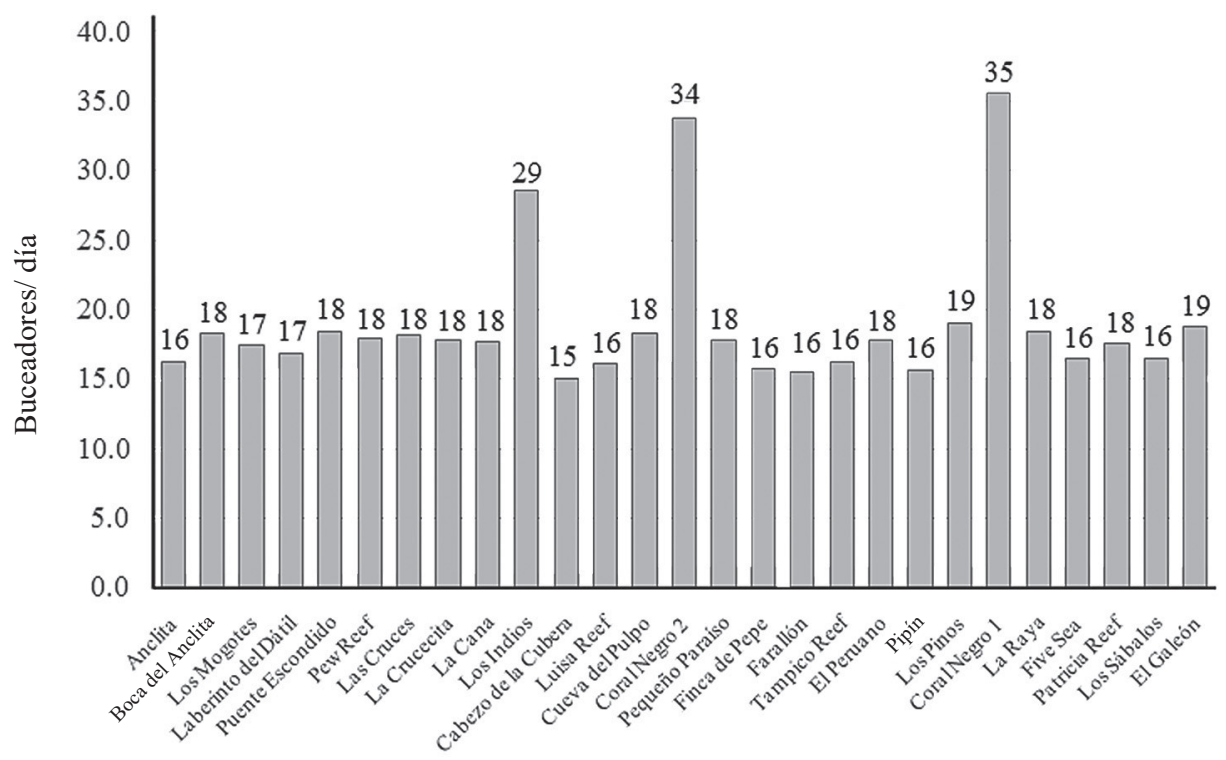

Sitios de buceo del Parque Nacional Jardines de la Reina

Fig. 4. Capacidad de carga efectiva de buceadores por sitios de buceo en el Parque Nacional Jardines de la Reina

Fig. 4. Effective carrying capacity of divers per diving sites in Jardines de la Reina National Park

los sitios mostró una condición entre "Buena" y "Muy Buena", con excepción de los sitios Cueva del Pulpo y Boca de Anclita, que manifestaron una condición "Regular". Referente a la densidad de colonias (densidad lineal de corales de más de $10 \mathrm{~cm}$ ) (crítica: $<4.0$, pobre: 5.00 7.99, regular: $8.00-11.99$, buena: $12.00-$ 16.00 y muy buena: $>16$ ), todos los sitios con excepción de Five Sea, reflejaron una condición "Crítica", al manifestar un valor menor a $4 \mathrm{col} / \mathrm{m}$.

Por su parte, según lo planteado por Alcolado \& Durán (2011), los sitios de las zonas con baja y media intensidad de buceo, ante el cubrimiento de corales vivos, manifestaron una condición entre "Buena" y "Regular" (7 de 10 sitios, o sea, el 70\% fue "Buena"). Ante la diversidad de corales pétreos, los sitios de la zona de baja intensidad de buceo mostraron una condición entre "Muy Buena" y "Buena", al igual que los sitios de la zona con media intensidad de buceo, con excepción del sitio La Cana, que manifestó una condición "Regular". Todos los sitios de ambas zonas mostraron una condición "Crítica" ante la densidad, con excepción del sitio La Raya. Mientras que los sitios de la zona sin buceo, ante el cubrimiento de corales vivos, manifestaron una condición "Regular". En cuanto a la diversidad de corales pétreos, una condición entre "Regular" y "Buena" y ante 
la densidad, una condición "Muy Buena".

Es válido destacar que en este trabajo se hace mayor énfasis en el indicador ecológico de cubrimiento de coral, ya que como plantean GonzálezDíaz et al. (2013), para un manejo adecuado de los arrecifes de coral es necesario conocer cómo y por qué ocurren cambios en dicho indicador a través de los años. Además de que la composición por especies, así como la densidad, son útiles como indicadores de riqueza y abundancia, facilitando la interpretación de los factores abióticos que regulan la comunidad. Por lo que se considera que el estado "crítico" que muestra el indicador de densidad en esta zona de estudio, puede estar dado por factores abióticos no analizados en el presente estudio. Por su parte, Connell et al. (1997) consideran que la mayor o menor diversidad está en dependencia de los disturbios que afectan a cada área arrecifal en particular. Sin embargo, los sitios de las zonas con alta, media y baja intensidad de buceo, ante este indicador, presentan una mejor condición que los sitios de la zona sin buceo. No obstante, existen otros indicadores ecológicos que, en su conjunto, definen la salud del arrecife y que tampoco fueron objeto de estudio en el presente trabajo.

Las condiciones de los sitios de buceo en el PNJR pueden deberse a varias razones. Primero, la cobertura, la diversidad y la densidad de corales son muy variables, por lo que podrían existir diferencias entre intensidades de uso que no son detectables.
Segundo, los sitios de buceo son ubicados en los lugares con mayor cobertura, diversidad y densidad de corales, lo que, de hecho, es uno de los criterios principales para que el turismo seleccione lugares como sitios de buceo. Tercero, el buceo autónomo en el PNJR, en apariencia, no está influyendo de forma negativa sobre las condiciones del arrecife coralino, específicamente, sobre los indicadores ecológicos de cubrimiento, diversidad y densidad de los corales pétreos. Los autores de este trabajo se inclinan por esta última explicación.

El hecho de que la mayoría de las infracciones cometidas hayan sido intencionales, al ser realizadas buscando posiciones adecuadas para tomar mejores fotos, muestra lo contrario a lo expuesto por Gallo et al. (2002) en San Andrés, Colombia, donde la cantidad de toques involuntarios resultó mayor a la de los toques voluntarios. Por su parte, el menor porcentaje de infracciones fue cometido por mujeres, lo que también apreciaron Rouphael \& Graeme (2001) en el Parque Marino de la Gran Barrera Arrecifal de Australia.

Los resultados, en general, coinciden, además, con los obtenidos por Hernández-Fernández et al. (2008) para los sitios de buceo de Cayo Coco, donde también el mayor número de contactos lo recibieron las gorgonias y la parte del equipo de buceo con que más se contactó a los organismos del arrecife coralino fue con las aletas. En dicho estudio, asimismo, estas infracciones fueron favorecidas por el empleo de 
cámaras subacuáticas. Rouphael \& Graeme (2001), en el estudio realizado en el Parque Marino de la Gran Barrera Arrecifal de Australia, consideraron que aquellos buceadores que portaban cámaras subacuáticas causaron un mayor daño al arrecife que aquellos que no portaban. Resultados similares, igualmente, fueron expuestos por Gallo et al. (2002) en San Andrés, Colombia. Por su parte, en un estudio realizado en el Parque Nacional Cabo Pulmo, Baja California, México, los corales fueron los organismos bentónicos que mayor porcentaje de impactos recibieron $\mathrm{y}$, de la misma manera, las aletas fueron el medio de contacto más frecuente (Álvarez, 2012).

$\mathrm{La}$ frecuencia de toques/min. fue alta si se compara con las detectadas por otros autores. Hernández-Fernández et al. (2008) determinaron, para los arrecifes de Cayo Coco, 2.9 toques/ min. en $12 \mathrm{~m}$ de profundidad y 1.5 toques $/ \mathrm{min}$. en $24 \mathrm{~m}$, profundidades similares a las tenidas en cuenta en el presente estudio y que se corresponden, igualmente, con zonas de escarpes poco profundos. Aquí los buceadores también se consideraron "con experiencia". Barker \& Roberts (2004) en Santa Lucía observaron entre 0.02 y 0.69 toques/ min., indistintamente del empleo de cámaras subacuáticas o no. Asimismo, se considera alta ante lo expuesto por Gallo et al. (2002) para los fotógrafos (1.35 toques/min.). Zakai \& ChadwickFurman (2002) en los arrecifes coralinos de Eilat, al norte del Mar Rojo (visitados al año por más de 250 000 buceadores, en solo $12 \mathrm{~km}$ de línea de costa), detectaron entre 0.25 y 0.55 contactos/min., independientemente de la topografía del arrecife, mientras que los daños mecánicos significativos fueron de alrededor de 0.08 daños mecánicos/min. (rompimiento) y la resuspensión de sedimentos se comportó en 0.19 resuspensión de sedimentos/min. No obstante, estos trabajos fueron realizados hace más de 7 años, tiempo en el que el uso de cámaras subacuáticas ha crecido sustancialmente por el abaratamiento de sus precios y, por tanto, cabría esperarse que en esos sitios las incidencias de los buceadores, con cámaras, hayan ascendido. También hoy existe una mayor accesibilidad al entrenamiento $\mathrm{y}$, de esta manera, se incrementa la facilidad de obtener las certificaciones de buceo.

Se obtuvo información anecdótica de que con el incremento del número de buceadores con cámaras subacuáticas, han aumentado las incidencias de los buceadores sobre los arrecifes coralinos del PNJR (N. López-Fernández, comunicación personal, octubre 23, 2014), por lo que la afectación al arrecife podría ser mayor. Se deben impartir charlas educativas a los grupos de buceadores en la bienvenida y antes de cada inmersión, así como tener una mayor supervisión durante el buceo para incidir mejor en la disminución de los contactos de los buceadores con los organismos del arrecife. También se impone la realización de investigaciones para determinar las consecuencias que pueden generar los contactos de los buceadores sobre los organismos. Sobre 
esta base se recomienda monitorear aquellos organismos que recibieron daños mecánicos (rompimiento), con el fin de conocer con precisión su evolución ante esta incidencia de los buceadores. Debe aumentarse, además, el número de buceadores observados durante la actividad.

El promedio total de buceadores/ sitio/día en el PNJR, así como el anual, fue mucho menor al reportado por Zakai \& Chadwick-Furman (2002) para los arrecifes de Eilat, al norte del Mar Rojo (30 000 buceadores poraño en lamayoría de los sitios), al reportado por Gallo et al. (2002) para los sitios de buceo más frecuentados en San Andrés (29 buceadores/sitio/día y 10658 anuales), quienes argumentan que el límite de visitantes no constituye en sí mismo un fin, sino un medio (y no el único) para proteger los recursos del área, así como al obtenido por Álvarez (2012) para el Parque Nacional Cabo Pulmo, Baja California, México(94380 anuales). Según Gallo et al. (2002), estos resultados deben ser revisados periódicamente, conforme las condiciones ambientales varíen, pues la disminución de la frecuencia de toques o el incremento en la capacidad de manejo pueden incrementar la capacidad de carga, acercándose a la capacidad de carga física (CCF) en la medida que los efectos tiendan a cero. Esta es una de las razones por las cuales los resultados y las conclusiones del presente estudio solo deben aplicarse a los sitios de buceo investigados, y no deben ser extrapolados a otros.

En un análisis más detallado por sitios de buceo, se pudo apreciar que de los 27 sitios, 24 muestran una Capacidad de carga efectiva (CCE) con valores que oscilan entre 16 y 19 buceadores/sitios/ días, valores que se corresponden con una capacidad de carga de intensidad de buceo entre media (16) y alta o máxima (19). Solo tres sitios muestran una CCE por encima de lo establecido como intensidad alta o máxima para las áreas protegidas cubanas (Perera et al. 2007). Teniendo en cuenta los criterios de estos autores, se sugiere que aquellos sitios donde el valor fue mayor o igual a 19 , pueden tener una intensidad alta de buceo (19 buceadores/sitio/día: Coral Negro 1, Coral Negro 2, Los Pinos y Los Indios), donde el valor fue mayor a 16 y menor a 19 , una intensidad media de buceo (16 buceadores/sitio/día) y los de menor a 16, una intensidad baja de buceo (12 buceadores/sitio/día) (Fig. 4).

\section{CONCLUSIÓN}

$\mathrm{La}$ incidencia del buceo autónomo en el PNJR, en apariencia, no está influyendo de forma negativa sobre las condiciones del arrecife coralino, específicamente, sobre el cubrimiento, diversidad y densidad de los corales pétreos. No obstante, con base en estos resultados y en los análisis sobre la capacidad de carga de cada sitio de buceo, el manejo de estos se debe enfocar en cumplir con el número de buceadores/sitio/día que se sugiere en este estudio.

\section{AGRADECIMIENTOS}

Los autores agradecen a Roy Phillips López, Evelio Alejandro Alemán y Omar J. Fernández Pérez del Centro de Investigaciones de 
Ecosistemas Costeros (CIEC), a la tripulación de la embarcación "La Reina" y a Noel López Fernández, Eduardo del Sol Cruz y Jorge L. Casonova de la Sucursal Marlin Jardines de la Reina, por sus valiosos aportes. Y, asimismo, a los evaluadores.

\section{BIBLIOGRAFÍA}

AGRRA. (2000). The AGRRA Rapid Assessment Protocol. Atlantic and Gulf Rapid Reef Assessment Methodology. Recuperado en junio 25, 2001, disponible en http:// coral.aoml.noaa. gov/agra/methodhome.htm

Alcolado, P. M. (1981). Zonación de los gorgonáceos someros de Cuba y su posible uso como indicadores comparativos de tensión hidrodinámicos sobre los organismos del bentos. La Habana. Inf. Cient. Téc. Inst. Oceanol., 187, 1-43.

Alcolado, P. M. \& Durán, A. (2011). Sistema de escalas para la clasificación y puntaje de condición del bentos e ictiofauna de arrecifes coralinos de Cuba y del Gran Caribe. Ser. Oceanol., 8, 25-29.

Álvarez del Castillo, P. (2012). Capacidad de carga de buceo del Parque Nacional Cabo Pulmo, Baja California, México. Trabajo de Grado no publicado, Instituto Politécnico Nacional. Centro Interdisciplinario de Ciencias Marinas. CICIMAR-IPN. Baja California, México.

Appeldoorn, R. S. \& Lindeman, K. C. (2003). A Caribbean-wide survey of marine reserves: spatial coverage and attributes of effectiveness. Gulf. Caribb. Res., 14, 139-154. doi: 10.18785/gcr.1402.11

Barker, N. H. L. \& Roberts, C. M. (2004). Scuba diver behavior and the management of diving impacts on coral reefs. Biol. Conserv., 12, 481-489. doi: 10.1016/j.biocon.2004.03.021
Caballero, H., Alcolado, P. M., González, P., Perera, S. \& Hernández-Fernández, L. (2013). Protocolo para el monitoreo de bentos en arrecifes coralinos. Versión ajustada a partir del método de campo AGRRA 2000. La Habana, Cuba: Centro Nacional de Áreas Protegidas.

Cederstav, A., Lawrence, J. \& Quintanilla, V. (Eds.) (2015). La Protección de los Arrecifes de Coral en México. Rescatando la Biodiversidad Marina y sus Beneficios para la Humanidad. IV Amenazas de los Arrecifes de Coral. AIDA. México, D. F.: Asociación Interamericana para la Defensa del ambiente.

Cifuentes, M., J-Mesquita, C. A. B., Méndez, M. E., Morales, N., Aguilar, D., Cancino, M. \& Turcios, M. (1999). Capacidad de carga turística de las Áreas de Uso Público del Monumento Nacional el Guayabo, Costa Rica. Turrialba, Costa Rica: WWF Centroamérica.

Connell, J. H., Hughes, T. P. \& Wallace, C. D. (1997). A 30 year study of coral abundance, recruitment and disturbance at several scales in space and time. Ecol. Monogr., 67, 461-488. doi: 10.1890/0012-9615(1997)067[0461:AY SOCA]2.0.CO;2

De la Guardia, E., Valdivia, A. \& GonzálezDíaz, P. (2004). Estructura de comunidades bentónicas en la zona de buceo de María la Gorda, ensenada de Corrientes, sureste de la península de Guanahacabibes, Cuba. Rev. Invest. Mar., 25, 103-111. Figueredo-Martín, T., Pina-Amargós, F., Angulo-Valdés, J. \& Gómez-Fernández, R. (2010). Buceo contemplativo en Jardines de la Reina, Cuba: caracterización y percepción sobre el estudio de conservación del área. Rev. Invest. Mar., 31, 23-32.

Figueredo-Martín, T., Pina-Amargós, F. \& Angulo-Valdés, J. (2013). Aportes 
de Bienes y Servicios Ambientales del Parque Nacional Jardines de la Reina (PNJR) a la economía del sector. En M. Arellano-Acosta (Ed.), Potenciando la conservación de la biodiversidad mediante la evaluación económica y ambientalmente sostenible de actividades productivas en el ecosistema Sabana-Camagüey, Cuba (pp. 85-105). La Habana, Cuba: Ministerio de Ciencia, Tecnología y Medio Ambiente. Proyecto PNUD/GEF Sabana-Camagüey.

Figueredo-Martín, T., Pina-Amargós, F., Angulo-Valdés, J., Ramírez-Roque, I. M. \& Pérez-Machado, A. M. (2014a). Vínculos entre el bienestar humano y servicios ambientales que prestan las áreas protegidas marinas del sur de Ciego de Ávila y Camagüey. En H. FerroAzcona, G. M. Gómez-País \& O. Acosta-Rodríguez (Eds.), Areas protegidas y comunidades humanas. Una mirada desde el Sur (pp. 159-189). La Habana, Cuba: Ministerio de Ciencia, Tecnología y Medio Ambiente. Proyecto GEFPNUD Archipiélagos del Sur.

Figueredo-Martín, T., Pina-Amargós, F., Hernández-Fernández, L., González de Zayas, R., Rodríguez-Cueto, Y., De la Guardia-Llansó, E. \& Angulo-Valdés, J. (2014b). Metodología para el Monitoreo de Uso Público en Áreas Marinas Protegidas (AMP) de Cuba. En E. MilianHernández (Ed.), Turismo sostenible en áreas marinas protegidas (pp. 112-123). La Habana, Cuba: Ministerio de Ciencia, Tecnología y Medio Ambiente.

Gallo, F., Martínez, A. \& Ríos, J. I. (2001). Capacidad de carga de visitantes en áreas de buceo de San Andrés Isla, Colombia, Pereira. Trabajo de Grado no publicado, Universidad Tecnológica de Pereira, Facultad de Ciencias Ambientales. Colombia. UTP.
Gallo, F., Martínez, A. \& Ríos, J. I. (2002). Efectos del buceo Scuba en fondos coralinos de San Andrés Isla, Colombia. Trabajo de Grado no publicado, Universidad Tecnológica de Pereira, Facultad de Ciencias Ambientales. UTP.

González-Díaz, P., Martínez-Rodríguez, Y. B., Perera-Pérez, O. \& Álvarez-Fernández, S. (2013). Estimación de indicadores ecológicos a nivel de comunidad y población de corales hermatípicos en arrecifes con grado diferente de impacto. UCE Ciencia. Revista de Postgrado, 1, 1-21.

González-Ferrer, S. (2009). Diversidad de Organismos. Celenterados-Filo Cnidaria: Clase Anthozoa, Subclase Zoantharia, corales pétreos, Orden Scleractinia. En R. Claro (Ed.), Biodiversidad marina de Cuba (pp. 42-46). La Habana, Cuba: Instituto de Oceanología.

Hernández-Fernández, L., RodríguezÁvila, L., Monticone, K. \& De la Guardia-Llansó, E. (2008). Incidencias del buceo recreativo sobre los arrecifes coralinos en Cayo Coco, Cuba. Rev. Invest. Mar., 29, 205-212.

Hernández-Fernández, L., Guimarais, M., Arias, R. \& Clero, L. (2011). Composición de las comunidades de octocorales y corales pétreos y la incidencia del blanqueamiento del 2005 en Jardines de la Reina, Cuba. Rev. Mar. Cost., 3, 77-90.

Kramer, P. (2003). Synthesis of coral reef health indicators for the western Atla. ntic: Result of the AGRRA program (1997-2000). En J. C. Lang (Ed.), Status of Coral Reefs in the Western Atlantic: Results of initial surveys, Atlantic and Gulf Rapid Reef Assessment (AGRRA) Program. Washington, D. C. EE. UU.: Board. 496, 1-42. doi: 10.5479/ si.00775630.496-3.1

Linton, D. R., Smith, R., Alcolado, P. M., Hanson, C., Edwards, P., Estrada, R., ...\& 
Wiener, J. (2002). Status of Coral Reefs in the Northern Caribbean and Atlantic Node of the GCRMN. En C. R. Wilkinson (Ed.), Status of coral reefs of the world: 2002. Global Coral Reef Monitoring Network (pp. 277-302). Townsville, Australia: Australia Institute of Marine Science.

McField, M. \& Kramer, P. (2007). Healthy Reefs for Healthy People: A Guide to Indicators of Reef Health and Social Well-being in the Mesoamerican Reef Region. Miami, FL, EE. UU.: Smithsonian Institution.

Miththapala, S. (2008). Coral Reefs. Coastal Ecosystems Series. Colombo, Sri Lanka: Ecosystems and Livelihoods Group Asia, IUCN.

Perera-Valderrama, S., Estrada, R., Hernández-Ávila, A., García-Sáez, J. C., Alcolado-Méndez, P. \& García-Rivas, M. C. (2007, noviembre). Metodología para la Determinación de la Capacidad de Carga de Visitantes, en las Áreas Marinas Protegidas de Cuba. Ponencia presentada en $58^{\text {th }}$ Gulf and Caribbean Fisheries Institute. Texas, EE. UU.

Pina-Amargós, F., Hernández-Fernández, L., Clero, L. \& González-Sansón, G. (2008). Características de los hábitats coralinos en Jardines de la Reina, Cuba. Rev. Invest. Mar., 29, 225-237.

Rouphael, B. \& Graeme, J. I. (2001). Take only photographs and leave only footprints?: An experimental study of the impacts of underwater photographers on coral reefs dive sites. Biol. Conserv., 100, 281-287. doi: 10.1016/ S0006-3207(01)00032-5

Sousa-Melo, R., Crispim, M. C., Rodriguez-Viana de Lima, E. \& KioharuNishida, A. (2006). Estimativa da capacidade de carga recreativa dos ambientes recifais da Praia do Seixas (Paraíba-Brasil). Turismo-Visão e Ação, $8(3), 411-422$.

StatSoft. (2004). STATISTICA for Windows. Computer program manual. Tulsa, OK: StatSoft, Inc.

Wilkinson, C. R. (2000). Status of coral reef of the world: 2000. Global Coral Reef Monitoring Network. Townsville, Australia: Australia Institute of Marine Science.

Zakai, D. \& Chadwick-Furman, N. E. (2002). Impacts of intensive recreational diving on reef corals at Eilat, northern Red Sea. Biol. Conserv., 105, 179-187. doi: 10.1016/ S0006-3207(01)00181-1

Zar, J. H. (1996). Biostatistical analysis (3ra. ed.). New Jersey, EE. UU.: Prentice-Hall, Inc.

Zlatarski, V. N. \& Martínez-Estalella, N. (1980). Escleractinios de Cuba con datos sobre sus organismos asociados (en ruso). Sofía, Bulgaria: Editorial Academia de Bulgaria. 